\title{
THE MYSTERY OF THE CHROMOSPHERE
}

\author{
HAROLD ZIRIN \\ Big Bear Solar Observatory, Caltech, Pasadena, CA 91125, U.S.A.
}

(Received 4 September, 1996; in final form 1 October, 1996)

(Dedicated to Cornelis de Jager)

\begin{abstract}
We discuss many aspects of the solar chromosphere from an observational point of view, and show that most existing models are in direct contradiction to radio and eclipse measurements. We plead for attention to the actual observed radio temperatures and density gradients, as well as images of the chromosphere. We find that the chromosphere is not in hydrostatic equilibrium and suggest that the support is due to the tangled intranetwork fields.
\end{abstract}

\section{Introduction}

While Kees de Jager has mostly looked at solar flares in recent years, I learned a lot from his earlier work on chromospheres and especially from the meeting he organized in Utrecht for Minnaert's retirement. My felicitations and thanks for a lifetime of discussions and arguments.

The chromosphere is the least-well understood layer of the Sun's atmosphere. Until recent years, it could only be detected fleetingly during a solar eclipse. Now it has become more accessible with radio observations, birefringent filters, and ultraviolet observations from space. However, each one of these has shortcomings which has left much of its behavior a mystery.

Part of the problem is that it is so dynamic and transient. At this height an ill-defined magnetic field dominates the gas and determines the structure. Since we do not know the physical mechanisms, it is impossible to produce a realistic model. Since most of the models ignored much of the data, they generally contradict the observational data. Typical models ignore other constraints and just match only the XUV data; this is not enough for a unique solution. It reminds one of the discovery of the sunspot cycle. While most of the great 18th century astronomers agreed that sunspot occurrence was random, only Schwabe, an amateur, took the trouble to track the sunspot number, thereby discovering the 11-year cycle (Johnson, 1857; Gleissberg, 1952).

The first information on the structure of the chromosphere came from the eclipse observations of the 1880's, which revealed the presence of strong unknown spectrum lines, a number of ionized lines, and a generally higher excitation spectrum than the photosphere, and the existence of emission lines, which were almost immediately explained by Kirchhoff. Quantitative measurements of the length of time it

(C) 1996 Kluwer Academic Publishers. Printed in Belgium. 
took for the moon to uncover the chromosphere gave us its height (about $5000 \mathrm{~km}$ ) which was an order of magnitude greater than expected in hydrostatic equilibrium. When the unknown lines were identified as the element helium and its excitation and ionization potentials discovered, it appeared that quite a high temperature was required to excite them. When the broad profiles of chromospheric $\mathrm{H} \alpha$ were observed, this reinforced the idea that the temperature was high. The problem of the great height of the chromosphere was a puzzle which attracted many papers during the 1930's.

When the high temperature of the corona was discovered, it was clear that somewhere in between we should see various stages of ionization corresponding to the range between the photospheric lines and the corona; therefore the ultraviolet spectrum was now predictable and was in fact predicted by Wooley and Allen (1948). Their prediction was generally borne out, except for the intensity of the lines.

There are major difficulties in observing the chromosphere. It must be seen against the tremendously bright photosphere. Only in radio and UV wavelengths does it appear as the solar image. One does see the chromosphere directly in the resonance lines $\mathrm{H} \alpha$ and $\mathrm{Ca}$ II $\mathrm{K}$, but special filters are required. Deviations from LTE make it practically impossible to interpret the intensity of the spectrum lines. While the photosphere is close to radiative equilibrium because of the high density and the fact that a few hundred kilometers down the atoms see a radiation field roughly the same as the local temperature, in the chromosphere the atoms look out into empty space and especially the lower-intensity lines are not formed anywhere near LTE. On the other hand, for many lines, especially the ultraviolet, self-absorption is not important and the total intensity of an emission line can be computed simply by the total integral of the collisional and radiative excitations which take place. That is, each of these processes leads to the emission of a photon. Further, while there is no lower limit to the non-LTE emission, the intensity cannot exceed the Planck function.

There was a great emphasis in the 1940's and 1950's on study of the flash spectrum. By taking very rapid exposures as the Moon covered and uncovered the chromosphere, it was possible to obtain quite good height resolution on the spectrum. It was hoped from the height gradients of these lines to determine the run of density, temperature and excitation in the chromosphere. This began with Menzel's landmark work in the Lick publications, the many HAO publications on the Khartoum eclipse, and many other works. However, this data was based on measurements with film and required differentiation of the film measurements, which resulted in really quite low accuracy and not a great deal of information. The main information gained was that in the low chromosphere the spectrum was dominated by neutral atoms and rare earths, but as we rose in height we found the spectral lines of ionized metals like strontium, calcium, and titanium and eventually above $2000 \mathrm{~km}$ the spectrum was dominated by the lines of hydrogen and the very strong $\mathrm{Ca}$ II resonance lines. Interestingly, the helium lines peak in intensity in 
the low chromosphere. They are practically absent at the lower heights, and then appear in a thin layer between 1000 and $2000 \mathrm{~km}$. Because the temperature was now known to rise to one million deg in the corona, the case for a hot chromosphere was strong.

\section{General Observational Constraints}

Because there are so many models and articles that clearly contradict the evidence, we should review that evidence. We will then discuss how one can form a picture that fits most of these. The most important observational constraints are as follows:

(A) In the chromosphere the temperature rises from the temperature minimum $(4000 \mathrm{~K})$ to that of the corona $(1000000 \mathrm{~K})$. However the 'transition region' is so thin as to be essentially invisible to radio waves, nor is there any marked limb brightening at any chromospheric wavelength (Simon and Zirin, 1969).

(B) The chromosphere exhibits a bright network in resonance lines, radio, and UV corresponding to the edges of the photospheric supergranulation. There is virtually an exact match between UV and radio maps of the chromosphere, as well as $\mathrm{K}$-line and $\mathrm{H} \alpha$ and magnetograms. Enhanced magnetic fields occur in the network. Both radio and UV measurements show a marked differentation between the network and the interior of the network cells. The latter may not have any temperature increase (certainly they never exceed $6000 \mathrm{~K}$, while the magnetic knots reach $16000 \mathrm{~K}$ at $3 \mathrm{~cm}$ (Gary, Zirin, and Wang, 1990)) and apparently rise to coronal temperatures. The mechanism of heating is, alas, unknown; but we know that it only occurs in the presence of a vertical diverging magnetic field.

(C) The appearance of the chromosphere changes markedly from center to wing of $\mathrm{H} \alpha$ (Figure 1), and it is still different in the He lines. While it appears as an irregular opaque limb band in $\mathrm{H} \alpha$ and $\mathrm{Ca} \Pi \mathrm{K}$, the wing of the line exhibits a thin forest of spicule jets which are found on the disk to occur only at the edges of the network. The spicule forest is far from opaque, but the centerline chromosphere is opaque in $\mathrm{H} \alpha$ and $\mathrm{K}$. Spicules show velocities up to $40 \mathrm{~km} \mathrm{~s}^{-1}$, and temperatures similar to the chromosphere. The average spicule height is lower than the general chromosphere as seen in centerline, but high macrospicules are observed at the pole. In the centerline, arches and fibrils abound, testifying to magnetic support. The D3 chromosphere is a thin band between 1000 and $2000 \mathrm{~km}$, much lower than the $\mathrm{H} \alpha$ chromosphere. Spicules are rather faint in the He lines.

(D) The brightness scale height of the chromosphere is between $500 \mathrm{~km}$ (Weart, 1972) and $1200 \mathrm{~km}$ (Ewell et al., 1993). The height of the chromosphere in $\mathrm{H} \alpha$ is about $5000 \mathrm{~km}$ in the line center, about $500 \mathrm{~km}$ higher (Johannesson and Zirin, 1996) at the poles (possibly due to the presence of coronal holes). The height in He D3 and 10830 (Jones and Duvall, 1996) is much lower about $1500-2000 \mathrm{~km}$.

(E) The gas flowing through the chromosphere is filtered so that elements of high first ionization potential are underabundant by a factor 10 . 


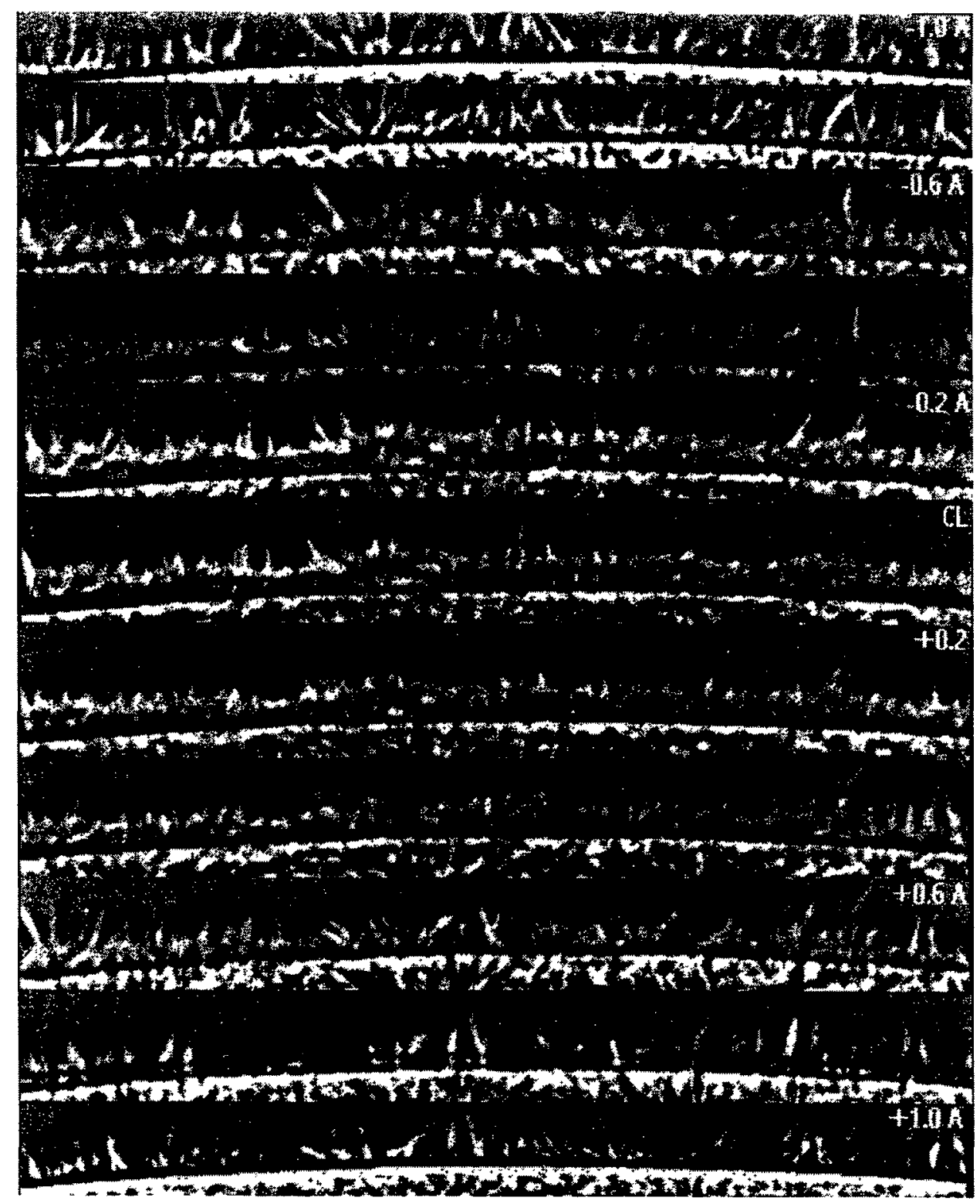

Figure 1. A series of $\mathrm{H} \alpha$ images ranging from $\mathrm{H} \alpha-1 \AA$ to $\mathrm{H} \alpha+1.0 \AA$ in steps of $0.2 \AA$. This region is about $20^{\circ}$ from the $\mathrm{N}$ pole. The images are taken sequentially about $1 \mathrm{~min} 20 \mathrm{~s}$ apart; however, intervening frames exist for evolution studies. The linewidth of each spicule, even allowing for the time interval, is surprisingly small. The general chromosphere is seen between $\mathrm{H} \alpha \pm 0.6 \AA$. The spicules clearly do not form an opaque wall.

(F) The slope of the Lyman continuum corresponds to a free electron temperature of $5000 \mathrm{~K}$, while the brightness is somewhat less. Therefore the electron temperature where $\mathrm{Ly}_{\mathrm{c}}$ is formed is $5000 \mathrm{~K}$.

(G) The radio temperature of the chromosphere in the $\mathrm{mm}-\mathrm{cm}$ range is between $6000 \mathrm{~K}$ and $8000 \mathrm{~K}$. This is the true electron temperature at $\tau=1$ in those wavelengths, density $N_{e}$ is $10^{10}-10^{11} \mathrm{~cm}^{-3}$. No limb brightening is seen.

(H) The UV emission is very weak; it is particularly weak for lines from half-filled shells. 


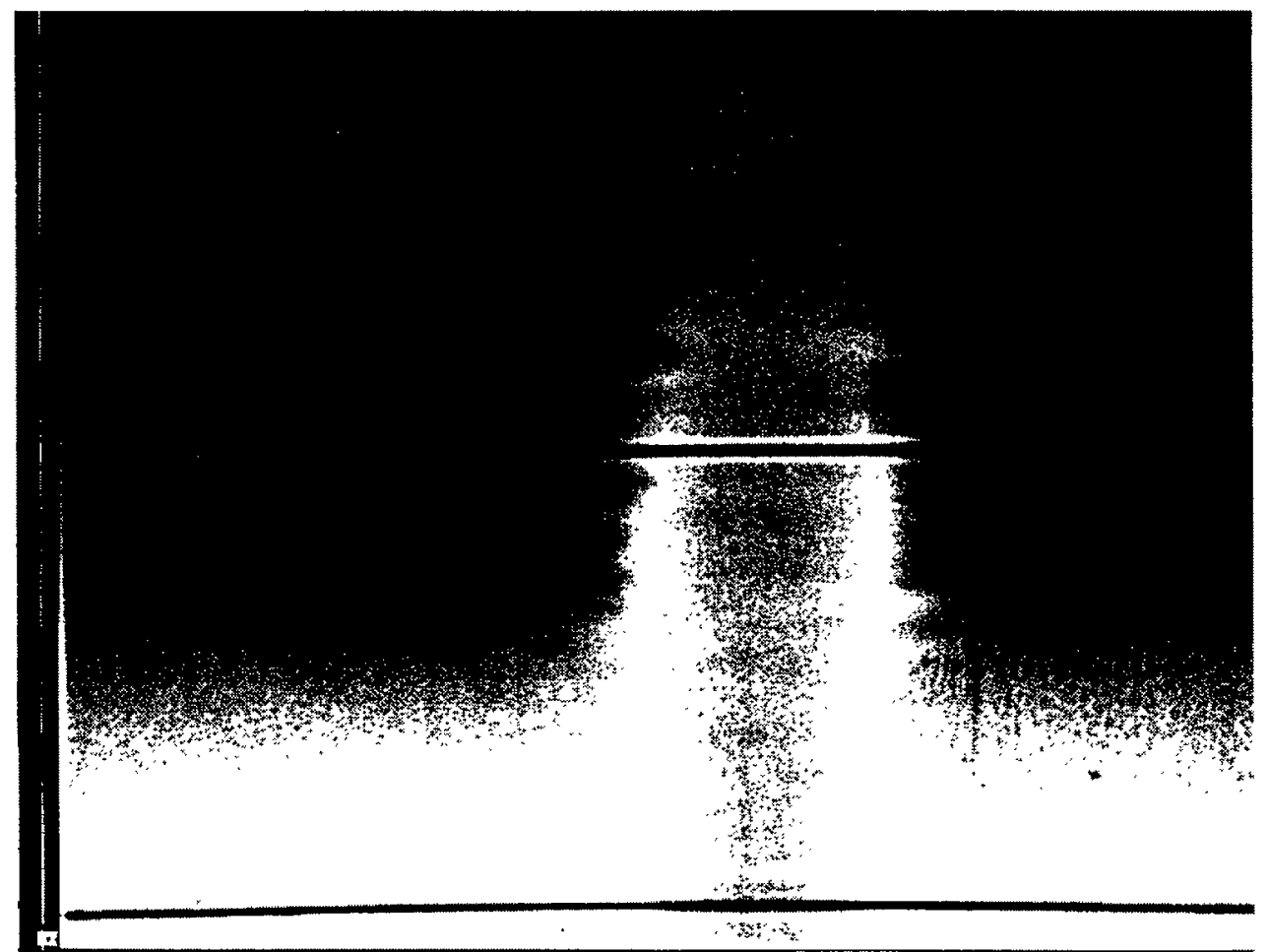

Figure 2. $\mathrm{H} \alpha$ spectrum of the chromosphere with a slit tilted $10^{\circ}$ from the limb to expand the vertical scale and sample different chromospheric regions. This image has been sharpened to bring out the spicule excursions on either side of the slit. The photosphere is at bottom.

Finally, we present two figures: Figure 1 is a set of modern images (taken within about $10 \mathrm{~min}$ ) of the limb chromosphere in $\mathrm{H} \alpha$. These images were made at steps of $0.2 \AA$ from $\mathrm{H} \alpha-1 \AA$ to $\mathrm{H} \alpha+1.0 \AA$ with the 65 -cm telescope at BBSO and then treated with a high-pass filter. The frames are separated by one minute in time. In the line center, we see that the chromosphere is unresolved up to about $5000 \mathrm{~km}$, where it suddenly breaks up into well-separated spicules extending a few thousand $\mathrm{km}$ higher. In the wings of the line, starting about $0.75 \AA$ from line center, the opaque chromosphere breaks up and spicules are seen right down to the photosphere, covering a fraction of the limb. The opaque band, which we shall refer to as the general chromosphere, is scarcely uniform, with multiple fine brightenings. The brightening at the top edge and the dark line at the base are artifacts of the filtering procedure. Some people have taken the position that the spicules merge to form an opaque chromosphere, and the general chromosphere ends at about a third the height of the layer seen here. However these images show no evidence of such strong convergence. In several of these frames, notably that in $\mathrm{H} \alpha+0.8 \AA$ ( $2 \mathrm{~d}$ from bottom), we can see dark spicules crossing the limb and appearing bright against the sky. Closer to line center, the spicule appears dark against the limb band. In some cases, the spicule may be brightened by its velocity relative to the surface, especially if it moves parallel to the surface (Zirin, 1969).

Figure 2 shows the $\mathrm{H} \alpha$ spectrum of the chromosphere. The slit has been placed at an angle of about $10^{\circ}$ to the tangent, so we could expand the height scale; the height 
of the spectrum is about $7000 \mathrm{~km}$. The horizontal dark lines are markers at the slit. In the lower half of the frame we see the photospheric spectrum. This spectrum has been artificially sharpened. The photospheric $\mathrm{H} \alpha$ is broadest at the limb, but the self-absorbed chromosphere may be even broader. The bright 'horns' are due to spicules, and can be seen to be due to many broad emission elements obscured in the line center but bright in the wings where they are solely prominent in Figure 1. Where the spicules are fast-moving, bright shifted streaks appear in the wings. This is verified by $\mathrm{H} \alpha$ slit jaw images. Above the middle marker, the spicules are fewer and less bright, and the horns disappear; a little higher, the general chromosphere becomes transparent, and we see only random Doppler-shifted spicules. Please bear in mind that each point in this spectrum refers to a different limb position as well as height.

\section{Radio Observations}

Because the radio temperature reflects the free-free emission, it measures the true electron temperature. The arguments over non-LTE effects, which often cloud such discussions, are irrelevant, because a Maxwellian distribution is unfailingly maintained. Radio waves can map the chromosphere by measuring the central brightness temperature at different wavelengths, by center-limb variation, by eclipse measures, and by detailed mapping.

\subsection{Central Brightness temperature}

If we look at the chromosphere with shorter and shorter wavelengths, we look at different depths. If we do this at sunspot minimum, we escape effects of active regions. Careful measurement of the central brightness temperature (Zirin, Baumert, and Hurford, 1991) shows a smooth transition in frequency between the optically thin corona and the opaque chromosphere. This model is well understood and confirmed by other radio observations. The chromosphere at $1 \mathrm{~cm}\left(N_{e}=10^{13} \mathrm{~cm}^{-3}\right)$ cannot exceed $8000 \mathrm{~K}$. I remember well a discussion with Shklovsky in 1961 where he cut short an argument over the high temperature of the chromosphere by simply pointing out this fact. What we see in the run of central brightness temperature is a coronal contribution gradually increasing with wavelength and a chromosphere at about $8000 \mathrm{~K}$ at all wavelengths. Many ponderous models can be found in the postwar literature that give temperatures of $20000 \mathrm{~K}$ or more. It was popular to simply ignore the radio data, the most dependable data we have.

\subsection{CENTER-LIMB VARIATION}

Center-limb variation is a powerful tool for studying height variation of temperature, as well as source distribution. Limb darkening enables us to measure the photospheric temperature gradient. Since the corona was hotter than the photosphere, early models of the solar radio emission predicted that there would be 
very strong limb brightening. The idea was that the temperature increases sharply upwards and so as we move toward the limb of the Sun the upper, hotter layers would be more visible and brighter. It was simply assumed this would be observed, as in the self-reversal of the He lines. A series of models were computed by Smerd which were dutifully reprinted in one publication after another with limb peaks two or three times brighter than the center of the disk. In fact, when Simon first brought the problem to my attention, it turned out that existing data showed no limb brightening at all (Simon and Zirin, 1969). Smerd was a great scientist, but this time he was wrong. Because the chromosphere is opaque to radio waves longer than a given frequency, we simply measure the temperature at $\tau=1$. Moving toward the limb is the same as observing the Sun center at a longer wavelength. So the flat response matches the flat frequency dependence; the chromospheric temperature varies little with height. The case in the UV is different, because the spectrum lines are optically thin. Therefore one would expect limb brightening even in the absence of temperature increase, simply due to the secant increase of path length. Although the intensity doubles at the limb, where we see the back side, the limb brightening inside the limb is minimal, except for lines like Ne VII, formed in the abrupt transition between chromosphere and corona. From the huge limb brightening in He D3, we know that a uniform layer does brighten sharply when viewed edge on. Similarly, X-ray images show limb brightening simply due to the increased path length. The answer probably lies in the weakness of the UV emission (the brightness temperature is only $4000 \mathrm{~K}$ ); there is so little transition region. The presence or absence of limb brightening could be determined by very accurate measurements of the total emission from the eclipsed Sun as the Moon crossed the chromosphere.

\subsection{ECLIPSE MEASUREMENTS}

Coates, Gibson, and Hagen (1959) made the first millimeter eclipse observations at Khartoum and determined that there was no limb brightening and the temperature did not exceed $8000 \mathrm{~K}$. But the Smerd cartoons, like the VAL (Vernazza, Avrett, and Loeser, 1976) model today, continued in the catechism. The belief was so deep that the observational result was assumed. In fact, only the most sensitive measurements have detected a small limb brightening, and only at wavelengths longer than the millimeter range $\left(N_{e}=10^{11} \mathrm{~cm}^{-3}\right)$.

Subsequent progress was made possible by telescope improvements and a technique introduced by Lindsey et al. (1983). While one could track the limb as the Moon covered it, one never knew exactly where in the beam it was. Using telescopes with good pointing, he continuously scanned the limb so he could instantaneously determine the position and the true flux.

The most accurate measurement of the chromospheric height gradient was performed at $850 \mu$ by Ewell et al. (1993) at the 1991 total eclipse, giving a height of $4700 \mathrm{~km}$ and scale height of $1000 \mathrm{~km}$ for the electrons. Measurement by 
other workers at various eclipses and wavelengths correspond well to this result. The CICM model (Table 5), presented in that paper, fits the radio data for all millimeter and centimeter wavelengths. It only measures $N_{e}, N_{i}$, and cannot see the chromosphere at heights where densities exceed $10^{10-11} \mathrm{~cm}^{-3}$. It certainly gives an accurate picture of the gradient of $N_{e} N_{i}$ in the middle and upper chromosphere. Further, the observations show an absolutely linear decrease in total brightness of the crescent; there is no change in temperature with height.

\subsection{MAPPING}

By using neighboring frequencies to add information, it has been possible to map the brighter elements of the chromosphere with high resolution at the VLA (Gary, Zirin, and Wang, 1990). The images, not surprisingly, match the K-line and magnetic fields. The magnetic network is bright, with a temperature of the order $16000 \mathrm{~K}$, while the major part of the chromosphere in between the network is barely detectable: less than $7000 \mathrm{~K}$. These maps are difficult because, in contrast to flares where a few bright sources appear, the chromosphere is a complex extended source of low brightness, and aperture synthesis must continue for hours to produce good maps, and then only in regions of enhanced network.

This point tells us that there are really two chromospheres, the intranetwork, where there is very little upward temperature increase, and the network, where the transition to the corona occurs. How the transition to corona occurs in the intranetwork region without an apparent temperature increase is unknown.

\section{The Problem of the Height Gradient}

\subsection{OPTICAL OBSERVATIONS}

Years ago the journals were filled with discussions of 'the height of the chromosphere'. It was clear that the apparent scale height of $1000 \mathrm{~km}$ far exceeded that in hydrostatic equilibrium. In modern times a convenient solution has been found denial. Although anyone can measure its height with a ruler and find it extending to $5000 \mathrm{~km}$, most publications state that it becomes corona at $2000 \mathrm{~km}$ above the surface. We cannot explain the great height or the erroneous models.

The understanding of the chromosphere has been hampered by the wide acceptance of the VAL (Vernazza, Avrett, and Loeser, 1976) model, a model fitting the photospheric data well, but extrapolating to the chromosphere on the assumption of hydrostatic equilibrium. This would be reasonable for a region of uniform height and structure that did not filter out neutral atoms. In any event it does not correspond to observational fact. Like Smerd's graphs, this model has hung on out of inertia, with no supporting chromospheric data. The great extension of the chromosphere is usually attributed to spicules, which are supposed to be what we see at the limb. But all observational data (Figure 1) show that spicules are lower than the general 
chromosphere, and there are not enough to obscure the sky. The eclipse data measure everything, showing a high density and low temperature up to $5000 \mathrm{~km}$, and are generally ignored. It must always be remembered that the Sun is not a workstation, but a real star.

The height of the chromosphere varies; for example, the $\mathrm{H} \alpha$ height gradient (Johannesson and Zirin, 1996) is found to be less (i.e., the chromosphere extends higher) in the polar holes. This odd fact contradicts the idea that magnetic fields support the height; no support can be found in a vertical field. Measurements of element abundances in the higher-energy particles in the corona and in the solar wind show a great deficiency of the 'high FIP' elements, atoms of high first ionization potential, such as carbon, nitrogen, and oxygen.

Hydrogen and helium are not generally referred to as high FIP elements because of their great abundance. It has been proposed that, at the point where elements are singly ionized, namely the chromosphere, the ionized particles are accelerated outward or at least exhibit a much higher scale height than the neutrals, which are not supported by the various magnetic fields which probably produce the extension of the chromosphere. This experimental fact alone confirms the deviation from hydrostatic equilibrium. When charged particles rise higher than neutral, there are special charge-sensitive means of support. We have found older coronagraph data show that the spectral lines of argon (with the exception of high-temperature lines in active regions) are absent from the normal quiet corona. This was another relic of the past; a line of argon X from the quiet corona at $5539 \AA$ had crept into the literature. Eclipse measurements dutifully recorded the lines as 'possible'. In fact they were absent, and Edlén (1969) showed the right wavelength was 5533. But A XIV, a higher-temperature line, is quite strong in flare condensations (Zirin, 1964). This supports the idea that complex magnetic fields and continuous ejection mechanisms are active in supporting the high scale height. These forces do not act on the neutral high-FIP elements.

It would be desirable to know the true height at which the corona begins, and probably this is the $5000 \mathrm{~km}$ value delivered by the eclipse data. But the scale height of the corona is large, and it projects against the disk so that it is impossible to determine its lower bound. While models place this at $2000 \mathrm{~km}$, the data say 5000. The transparency of the corona to $\mathrm{cm}$ waves set it at wherever the density falls to $10^{8} \mathrm{~cm}^{-3}$. Transition zone lines double in intensity when we peek over the limb, but because there is a long sight line in the corona ahead of the limb there is no sharp gradient. Radio eclipse measures at longer wavelengths yield data consonant with the millimeter data; there is little or no corona at the low altitudes.

Many models assume pressure equilibrium at the corona-chromosphere interface in the model. This may be, but probably is in error. Two gases of different temperature cannot have a stable equilibrium. If the pressure $N \sigma v^{2}$ is equal on both sides, the flux $N \sigma v$ differs by the square root of the temperature ratio and there is a rapid diffusion into the hot side. Since this balance is imposed by external factors (or, simply, models), the actual run of density and temperature must take 
precedence over the putative theoretical values. We have powerful tools to do this, but it has not yet been done. Athay (1981) showed that a conduction model with downward heat flow would give a sharp lower boundary, but the dynamics would quickly wash it out.

What is the magnetic structure in the chromosphere? On the larger scale this is determined by the network elements. These fields emerge radially on the average but show significant divergence as soon as they emerge. We have measured these (Bass and Zirin, in preparation) by measuring how far onto the disk one may detect the apparent reversal of sign of longitudinal field (this effect may be seen on the polar magnetograms displayed on the BBSO home page, http://bigbear.caltech.edu). The divergence is $\pm 15^{\circ}$. Thus the field lines converge over the network cell, but at this low angle, the field lines only enter a few thousand $\mathrm{km}$ before they are out of the chromosphere. Where fields are stronger, as in the enhanced network, the tilt may be as high as $60^{\circ}$ (Suematsu, Wang, and Zirin, 1995), but that is unusual. So the fields are determined by the intranetwork (IN) fields. We see rapidly moving small magnetic elements of both polarities inside the cells (Zirin, 1985). Their lifetimes are a few hours, and they must produce a tangled web of fields in the chromosphere which could play an important role in supporting the chromosphere. The high-FIP filtering, on the other hand, must be due to the hydromagnetic nature of spicules, which flow along field lines.

\section{Helium}

Understanding of the excitation of He has been another difficult question. Because the He excitation potential was so high, it was assumed that the chromosphere was quite hot. Further, the excitation temperature for singlet helium was determined by Athay and Menzel to be $20000 \mathrm{~K}$, although triplet He was found to be clearly excited by the photospheric radiation. Goldberg (1939) first proposed that chromospheric He is excited by coronal back-radiation; he was echoed by other authors and Zirin (1975) later developed formal calculations to establish this. A review of the observational facts makes calculation superfluous:

(a) While other strong lines fall off uniformly from photosphere upwards, the best-observed line, D3, peaks in a thin layer from 1000 to $2000 \mathrm{~km}$ above the surface. The line is 100 times stronger tangent to the limb than on a radial line. With good seeing, a dark band appears below the He layer and the top of the layer is $2000 \mathrm{~km}$ below the top of the $\mathrm{H} \alpha$ chromosphere.

(b) All helium lines are greatly weakened under coronal holes.

(c) The $584 \AA$ A resonance line displays a narrow central reversal (Ogawa, Phillips, and Judge, 1984; Phillips, Judge, and Carlson, 1982), characteristic of radiationexcited lines. Some publications (e.g., Fontenla, Avrett, and Loeser, 1993) have simply ignored this data and assumed that observations confirmed their requirements. 
Calculation shows the He forms a classic Chapman layer, where the UV radiation from above (as in the ionosphere) diminishes by absorption as we go downward, till the increased neutral component snuffs it out. The radiation in the $\lambda 304$ line of He II plays an important role. The He atoms are photoionized by the UV flux, and those atoms landing in metastable triplet $\mathrm{He}$ I states are excited thence by photospheric radiation. For some reason, this result enraged the theoreticians. Milkey and Heasley (1976) correctly pointed out that a deep self-absorption would have to be found, assuming it would not. When Phillips, Judge, and Carlson (1982) found the reversal, it was ignored. The arguments for photoexcitation of helium are largely phenomenological - coronal hole effects and the low height of the $\mathrm{He}$ chromosphere are strong arguments for photoionization. The theoreticians, deep in their workstations, place more faith in models and calculations, which may fit their view if not too many observational factors are introduced. This episode brings out a fundamental difference in philosophy.

\section{Spicules}

Spicules are a dramatic aspect of the chromosphere which have been accused of a multitude of ills, such as the source of the solar wind or the coronal heating. They were seen quite early by the Italian observers (for example, described by Pringsheim (1910), and rediscovered by Roberts (1945)). Because the velocities and Doppler shifts of spicules are high, they are best seen in the wing of $\mathrm{H} \alpha$ at the limb, where they are slightly brighter than the disk centerline. On the disk, however, they are generally (but not always) in absorption in centerline and always in absorption in the wings. They are seen as clusters of jets radiating from the bright magnetic elements of the network. If those fields are weak, as in regions where polarities are mixed and there are no plage remnants, the spicules are short and randomly oriented. Where the network fields are strong, as in the enhanced network, the spicules are elongated and follow the local field lines. There are no spicules in plages, but they are quite prominent on the edges of plages. Cragg, Howard, and Zirin (1963) were able to identify them on the disk because near the limb they always appear on the limbward side of the network elements, showing that they lie above. There are three classes of spicules:

Normal spicules are ejected from magnetic network elements all over the disk with velocities up to $40 \mathrm{~km}$ (Suematsu, Wang, and Zirin, 1995). They typically reach to $4000-5000 \mathrm{~km}$. They are somewhat longer in active regions, do not appear above plages, and always fall back to the surface.

Macrospicules are concentrated at the poles (10 times more frequent there) and do $n o t$ fall back to the surface (at least as seen in $\mathrm{H} \alpha$ ). They extend up to $20000 \mathrm{~km}$ in $\mathrm{H} \alpha$ and higher in $\lambda 304$.

Eruptive spicules do not show the thin, directed profiles of the other two classes and maybe should not be called that. But their $\mathrm{H} \alpha$ counterparts arise from the 
network like the others and shoot outward with somewhat higher velocities. They were discovered in C IV by the NRL imaging spectrograph. We do not have enough data to be completely confident these are the same as the $\mathrm{H} \alpha$ events.

While normal spicules appear to flow along a flux tube in approximately ballistic fashion, the second two certainly do not. The 'tube' itself plays a role, and the great extent of macrospicules suggests that processes along the path accelerate the outward flow. The eruptive events expand or explode laterally.

Spicules have been proposed to explain the huge discrepancy between the observed and hydrostatc equilibrium. Like the emperor's new clothes, the theoretical chromosphere is hidden at the limb by a forest of spicules, which everyone agrees are non-hydrostatic. Unfortunately, none of this was backed up by observation. In the usual fashion, gedanken observations were introduced to prop up a desirable model. Unfortunately, the Sun does what it wishes. Examination of spicules seen on the disk, even in enhanced chromosphere, shows that they cover at most a few percent of the surface. Since the multiplication factor (ratio of tangential to radial coverage) for a $5000 \mathrm{~km}$ high forest is 30 for carefully placed spicules and only 6 if they are placed at random, the spicule forest does not present an opaque wall, but the general chromosphere does. As a simple glance at Figure 1 demonstrates, what we see at the limb is the chromosphere, with a scattering of spicules covering about a third of the limb and not even extending to the top of the chromosphere. It should be noted that the disk observations show that the spicules are no larger in centerline than in the $\mathrm{H} \alpha$ wings, where we count them.

\section{Ultraviolet Observations}

One would think that observations of the solar ultraviolet would solve many of the problems. However, the intensity of these lines was very much lower than expected and to this day images with adequate resolution have not been obtained. While the UV mimics the radio images, brightening in the network, it is impossible to tell if it comes from the spicules or the magnetic regions at their base. The lines show a deep minimum in intermediate ionization stages of $\mathrm{C}, \mathrm{N}$, and $\mathrm{O}$ (Zirin, Hinteregger, and Hall, 1963) and the brightness temperature in the extreme ultraviolet scarcely exceeds 4000 degrees. This gives a remarkable contradiction. Lines are observed of high ionization stages such as carbon 4 , neon 5 , oxygen 5 , which are only formed at temperatures of 100000 degrees or more but with brightness temperatures 20 times less. This led to a period of madly erroneous chromospheric models which assumed that a series of hot layers ranging in temperature from the 5000 degrees of the high photosphere to the million degees of the corona existed in a density coresponding to what one expected from hydrostatic equilibrium. Pottasch (1963) introduced a popular method to determine the emission measure at each temperature by assuming the lines always were emitted at the optimum temperature; Zirin and Dietz (1963) proposed a less popular method where the line ratios were used to 
Table I

CICM

\begin{tabular}{rlll}
\hline $\begin{array}{l}\text { Height } \\
(\mathrm{km})\end{array}$ & $\begin{array}{l}\text { Temperature } \\
(\mathrm{K})\end{array}$ & $\begin{array}{l}N_{e} \\
\left(\mathrm{~cm}^{-3}\right)\end{array}$ & $\begin{array}{l}N_{\mathrm{H}} \\
\left(\mathrm{cm}^{-3}\right)\end{array}$ \\
\hline 500 & 4410 & $3.01(+11)$ & $2.61(+15)$ \\
600 & 4620 & $2.25(+11)$ & $1.19(+15)$ \\
800 & 5220 & $1.90(+11)$ & $2.50(+14)$ \\
1000 & 5820 & $1.61(+11)$ & $5.95(+13)$ \\
1200 & 6410 & $1.36(+11)$ & $1.10(+13)$ \\
1400 & 6540 & $1.15(+11)$ & $7.37(+12)$ \\
1700 & 6620 & $8.98(+10)$ & $4.05(+12)$ \\
2000 & 6700 & $6.99(+10)$ & $2.22(+12)$ \\
2300 & 6780 & $5.45(+10)$ & $1.29(+12)$ \\
2600 & 6860 & $4.24(+10)$ & $6.69(+11)$ \\
2900 & 6940 & $3.30(+10)$ & $3.67(+11)$ \\
3200 & 7020 & $2.57(+10)$ & $2.01(+11)$ \\
3500 & 7100 & $2.00(+10)$ & $1.11(+11)$ \\
3800 & 7180 & $1.56(+10)$ & $6.07(+10)$ \\
4100 & 7260 & $1.21(+10)$ & $3.33(+10)$ \\
4400 & 7340 & $9.46(+9)$ & $1.83(+10)$ \\
4700 & 7420 & $7.37(+9)$ & $1.00(+10)$ \\
5000 & 7500 & $5.74(+9)$ & $5.50(+9)$ \\
\hline
\end{tabular}

estimate the emission temperature. Neither method allowed for the fact that the emission mainly comes from the network, introducing a large filling factor. The network source tends to explain the low limb brightening; we see a mix of bright network and dark intranetwork, and these have more or less the same ratio wherever we look.

Why cannot we just sort out the structure with images? In high-resolution movies the $\mathrm{H} \alpha$ chromosphere is a seething, oscillating mass of fibrils. While one cannot make order out of it, it does look like individual loops supported by magnetic fields. It certainly is not homogeneous.

\section{Conclusions}

We have criticized most of the models and therefore should offer something in exchange. This is very difficult. We already have set forth the CICM model (Table I). This gives an accurate description of the run of $N_{e} N_{i}$ with height. The difficulty comes with explaining why this is not in hydrostatic equilibrium. Very probably this is due to magnetic support. Certainly the element separation indicates this.

The IN (Zirin, 1985) elements are of mixed polarity and live only a few hours. The network elements are stronger and live for months. They diverge by $\pm 15^{\circ}$ at the photosphere. However, spicule tilts suggest that they diverge further in the 
chromosphere. Nonetheless the cells are so large $(30000 \mathrm{~km})$ that the network fields are not important through the cell. The IN elements, however, must fill the cell with horizontally stretched fields. Since they arise in N-S pairs (not proven or demonstrated, but we assume the absence of monopoles), they separate horizontally and drag the field lines out horizontally. The fields are complex because the IN elements merge and reconnect with the network when they reach the cell edges. This gives us just the sort of support that could produce the $1000 \mathrm{~km}$ scale height. This does not explain the variation of the chromospheric height with latitude.

\section{Acknowledgements}

I appreciate the help of Anders Johannesson who showed me how to carry out the high-pass filtering. This work was supported by NSF under grant ATM-9525971 and by NASA under Grant NAGW-1972.

\section{References}

Athay, R. G.: 1981, Astrophys. J. 249, 349.

Bass, J. and Zirin, H.: 1996, in preparation.

Coates, R. J., Gibson, J., and Hagen, E.: 1959, Astrophys. J. 128, 406.

Cragg, T., Howard, R., and Zirin, H.: 1963, Astrophys. J. 138, 303.

Edlén, B.: 1969, Solar Phys. 9, 439.

Ewell, M. W., Zirin, H., Jensen, J. B., and Bastian, T. S.: 1993, Astrophys. J. 403, 426.

Fontenla, J. M., Avrett, E. H., and Loeser, R.: 1993, Astrophys. J. 406, 319.

Gary, D. E. and Zirin, H.: 1988, Astrophys. J. 329, 991.

Gary, D. E., Zirin, H., and Wang, H.: 1990, Astrophys. J. 355, 321.

Gleissberg, W.: 1952, Die Haüfigkeit der Sonnenflecken, Leipzig.

Goldberg, L.: 1939, Astrophys. J. 89, 673.

Johannesson, A. and Zirin, H.: 1996, Astrophys. J., in press.

Johnson, M. J.: 1857, Monthly Notices Roy. Astron. Soc. 17, 126.

Jones, H. P. and Duvall, T.: 1996, Astrophys. J., to appear.

Lindsey, C., Becklin, E. E., Jefferies, J. T., Orrall, F. Q., Werner, M. W., and Gately, I.: 1983, Astrophys. J. 308, 448.

Milkey, R. and Heasley, H.: 1976, Astrophys. J. 210, 827.

Ogawa, H. S., Phillips, E., and Judge, D. L.: 1984, J. Geophys. Res. 89, 7537.

Phillips, E., Judge, D. L., and Carlson, R. W.: 1982, J. Geophys. Res. 87, 1433.

Pottasch, S. R.: 1963, Astrophys. J. 137, 945.

Pringsheim, E.: 1910, Physik der Sonne, Teubner, Leipzig.

Roberts, W. O.: 1945, Astrophys. J. 101, 136.

Simon, G. and Zirin, H.: 1969, Solar Phys. 9, 317.

Suematsu, Y., Wang, H., and Zirin, H.: 1995, Astrophys. J. 450, 411.

Vernazza, J., Avrett, E. H., and Loeser, R.: 1976, Astrophys. J. Suppl. 30, 1.

Weart, S.: 1972, BBSO preprint No. 0007.

Wooley, R. V. d. R. and Allen, C. W.: 1948, Monthly Notices Roy. Astron. Soc. 108, 292.

Zirin, H.: 1964, Astrophys. J. 140, 1216.

Zirin, H.: 1969, Solar Phys. 7, 243.

Zirin, H.: 1975, Astrophys. J. 199, L63.

Zirin, H.: 1985, Australian J. Phys. 38 (6), 961.

Zirin, H. and Dietz, R.: 1963, Astrophys. J. 138, 664.

Zirin, H., Baumert, B. M., and Hurford, G. J.: 1991, Astrophys. J. 370, 779.

Zirin, H., Hinteregger, H. E., and Hall, L. A.: 1963, in W. Priester (ed.), Space Research III, Amsterdam. 